\section{EL FUTURO DEL HÁBITAT: REPENSANDO LA HABITABILIDAD DESDE LA SOSTENIBILIDAD. EL CASO ESPAÑOL'}

Joaquim Arcas-Abella², Anna Pagès-Ramon ${ }^{3}$, Marina Casals-Tres ${ }^{4}$

\section{Resumen}

La capacidad de nuestras sociedades de alcanzar y mantener en el tiempo unas condiciones habitacionales coherentes con el derecho a un hábitat digno, está inevitablemente vinculada a la integración del reto de la sostenibilidad a los mecanismos de generación de nuestras viviendas, barrios y ciudades, pues depende directamente de los recursos disponibles y de la capacidad de emisión de residuos.

En base a este vínculo, se propone un despliegue del concepto de edificación sostenible, mediante la transposición de la definición de desarrollo sostenible formulada por el informe Bruntland, que permite establecer los eslabones que vinculan la satisfacción de necesidades humanas con el uso de recursos, revelando que la edificación sostenible debe abordar el cierre de los ciclos materiales en

\section{THE FUTURE OF HABITAT: RETHINKING HABITABILITY FROM SUSTAINABILITY. THE SPANISH CASE ${ }^{1}$}

Joaquim Arcas-Abella², Anna Pagès-Ramon³, Marina Casals-Tres ${ }^{4}$

\begin{abstract}
The ability of our societies to reach and maintain housing conditions consistent with the right to decent habitat is inevitably related to the integration of the sustainability challenge into the mechanisms for housing, neighborhood and city generation, since it directly depends on both available resources and the production of waste.
\end{abstract}

Based on this relation, the sustainable building concept is thoroughly explained through the transposition of the sustainable development definition proposed by the Bruntland report, which allows the linkage of human needs satisfaction with the use of resources. In this way, sustainable housing should address the 
la consecución de su objetivo principal: dotar de habitabilidad.

Bajo este enfoque, se analiza el concepto actual de habitabilidad en relación con el impacto ambiental, y argumenta la importancia de redefinirla como una demanda social de disponibilidad de las condiciones precisas para satisfacer las necesidades socialmente reconocidas; una nueva concepción de la habitabilidad que obliga a exceder el ámbito doméstico para abarcar la escala urbana, y que deviene variable para dar respuesta a las diferentes demandas sociales presentes hoy en día.

\section{PALABRAS CLAVE: HABITABILIDAD, VIVIENDA,} SECTOR DE LA EDIFICACIÓN, SOSTENIBILIDAD

Fecha de recepción: 05.03.11

Fecha de aceptación: 10.06.11

1 Proyecto de investigación "La redefinición de la habitabilidad", con fondos procedentes de los programas FI del Departament d'Economia i Coneixement de la Generalitat de Catalunya (20092012) y FPU del Ministerio de Educación y Ciencia del Gobierno de España (2005-2008), y con financiación puntual procedente del Ministerio de Medio Ambiente del Gobierno de España (2006-2007) y del Departament de Medi Ambient i Habitatge de la Generalitat de Catalunya (2009-2010). Una versión anterior de este trabajo fue presentada en el Congreso Internacional Sustainable Building 2010.

2 España. Personal docente, investigador Universitat Politècnica de Catalunya.Email: joaquim.arcas@upc.edu

3 España. Personal docente, investigadora Universitat Politècnica de Catalunya.Email: anna.pages@upc.edu

4 España. Personal docente, investigadora Universitat Politècnica de Catalunya.Email:marina.casals@upc.edu last phase of material cycles in order to reach its main goal: provide habitability.

Under this perspective, this paper analyzes the current concept of habitability in relation to the environmental impact and discusses the importance of redefining it as a social demand for the precise conditions to meet socially recognized needs. This new conception of habitability transcends the domestic domain to reach out urban spheres, thus generating variables to satisfy today's social demands.

\section{KEY WORDS: HABITABILITY, HOUSING, BUILDING SECTOR, SUSTAINABILITY}

\section{Received: 05.03.11}

Accepted: 10.06.11

1 Research project "Redefining Habitablity", funded by the FI program, Ministry of Economy and Knowledge, Generalitat de Catalunya (2009-2012); FPU program, Ministry of Education and Science (2005-2008); Ministry of the Environment (2006-2007) and Ministry of the Environment and Housing, Generalitat de Catalunya (2009-2010). A previous version of this research was presented in the Sustainable Building Congress 2010.

2 Spain. Academic Researcher. Architecture, Energy and Environment (AIEM), Polytechnic University of Catalonia Barcelona Tech (UPC). email: joaquim.arcas@upc.edu

3 Spain. Academic Researcher. Architecture, Energy and Environment (AIEM), Polytechnic University of Catalonia Barcelona Tech (UPC). email: anna.pages@upc.edu

4 Spain. Academic Researcher. Architecture, Energy and Environment (AIEM), Polytechnic University of Catalonia Barcelona Tech (UPC). email: marina.casals@upc.edu 


\section{Introducción}

El derecho universal a una vivienda digna y adecuada, entendida desde la perspectiva sistémica desarrollada por la Conferencia Hábitat I de Vancouver $(1976)^{5}$ y, posteriormente, por el Comité de Derecho Humanos de Naciones Unidas (1991) ${ }^{6}$, ha supuesto y supone todavía una de las principales reivindicaciones sociales de nuestra historia, por cuanto su consecución representa un punto crucial en el desarrollo vital de toda persona y de toda comunidad humana.

Es innegable que tras 35 años de la Declaración de Vancouver -y más aún respecto al origen de los asentamientos humanos- las condiciones de satisfacción de las necesidades de cobijo de la población mundial, aunque todavía lejos de ser aceptables, han evolucionado positivamente en términos generales, si bien el perentorio problema de desigualdad en la capacidad de desarrollo presente entre regiones y entre ciudadanos sigue sin tener respuesta.

El camino trascurrido hasta hoy esboza la dimensión y la dificultad de alcanzar el objetivo fijado por la Declaración Universal de Derechos Humanos; la multiplicidad de factores que congrega el hábitat humano ha requerido y continuará requiriendo numerosas acciones y esfuerzos proceden-

5 NACIONES UNIDAS. Primera Conferencia de Naciones Unidas sobre los Asentamientos Humanos,1976.

6 NACIONES UNIDAS. Comité de Derechos Humanos, 1991. tes de todos los agentes implicados en este proceso, desde las instituciones internacionales, hasta los propios moradores.

La complejidad de la tarea, en lugar de disminuir, presenta ahora un nuevo elemento a añadir a los tradicionales campos de batalla propios de la edificación: la ineludible exigencia de sostenibilidad de todas las actividades humanas.

La consolidación entre la comunidad internacional de la certeza de las afirmaciones que vienen siendo formuladas desde hace años acerca de la influencia negativa que ejerce la humanidad sobre el medio que nos sustenta, debería conllevar la adopción de la sostenibilidad como nuevo paradigma caracterizador del metabolismo socioeconómico.

El reto que representa la incorporación de la sostenibilidad al proceso de consecución de un hábitat adecuado mediante la acción edificatoria, en suma, de construcción de unos pueblos y ciudades capaces de albergar dignamente a sus habitantes, no debería ser considerado simplemente como una barrera más a superar al final una larga carrera.

Más bien al contrario, la demanda de sostenibilidad exige la revisión de la experiencia acumulada en torno al hábitat, ya que supone un reto transformador que de no ser abordado sistémicamente 
puede anular cualquier intento de mejora articulado desde las estrategias actuales y, más allá, puede significar a mediano plazo un retroceso en la capacidad de albergar a la comunidad humana.

En esta dirección, y como respuesta a la responsabilidad de continuar proveyendo el hábitat socialmente necesario, la planificación y la ejecución de la edificación debería ser enfocada desde el conocimiento de los nuevos condicionantes que impone la sostenibilidad y, en consecuencia, redefinida para posibilitar su viabilidad en el futuro.

\section{El reto de la sostenibilidad}

La actual crisis ambiental a la que se enfrenta la humanidad nació con la Revolución Industrial, al cambiar el sistema productivo orgánico, basado en un metabolismo circular -reintegrador de los residuos al proceso productivo- por el sistema productivo mineral, caracterizado por operar mediante un metabolismo lineal, que expele sus residuos fuera del sistema, al medio ${ }^{7}$ (Figura 1).

El cambio de metabolismo tiene una influencia directa sobre los impactos que generan los residuos. En el caso del metabolismo circular, propio de las sociedades orgánicas, los residuos son devueltos y absorbidos por el medio al mismo tiempo que permiten una restitución de los nutrientes necesarios

7 WRIGLEY, E. A., 1992. p. 110. para la conservación de la capacidad productiva de la biosfera que, de este modo, puede continuar produciendo la mayor parte de los recursos que estas sociedades precisan. Por el contrario, en el caso del metabolismo lineal, propio de las sociedades minerales o industrializadas, los residuos se envían y se acumulan en el medio, en forma de contaminación que degrada la biosfera que, al no formar parte del sistema productivo -ya que la mayor parte de los recursos se obtienen de la litosfera- no se percibe ya como un factor productivo indispensable.

Únicamente en el momento en el que aparece una conciencia social de que la degradación del medio causada por los residuos del metabolismo lineal pone en peligro -y en muchos casos de forma irreversible- los sistemas naturales que sustentan la vida, se genera una demanda de sostenibilidad que sirve como punto de partida de acciones que tienen como finalidad reducir el impacto ambiental del sistema productivo industrial, limitando, de forma progresiva, su capacidad contaminante.

A lo largo de los últimos años, el crecimiento de la demanda de sostenibilidad se ha traducido en un incremento del número y amplitud de estrategias y acuerdos contra la capacidad emisiva del sistema productivo. La lucha contra el cambio climático representa, en este sentido, el ejemplo más claro. Des- 
FIGURA 1. ESQUEMA DE LOS SISTEMAS PRODUCTIVOS ORGÁNICO Y MINERAL
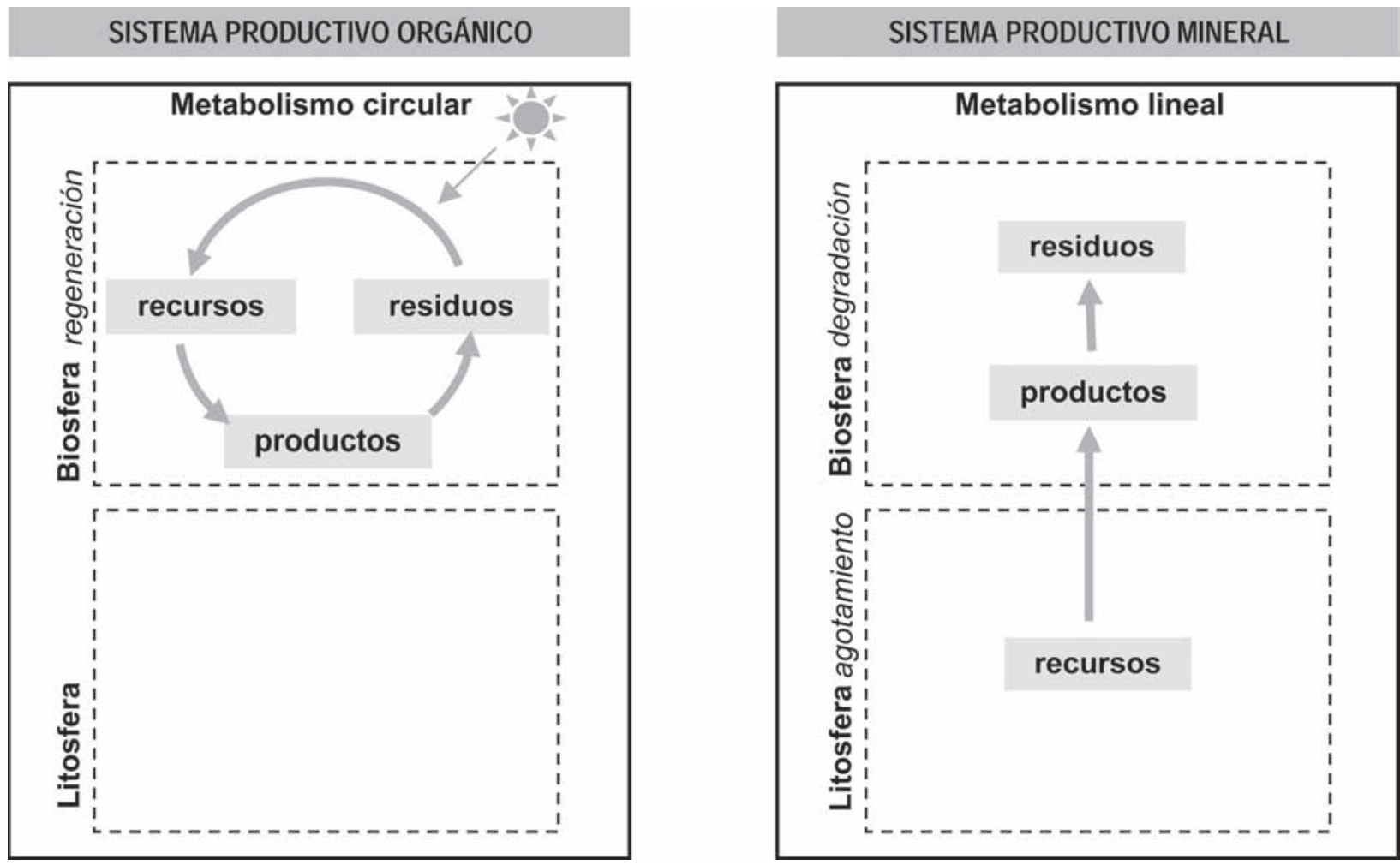
de hace varios años la comunidad internacional se ha preocupado por el impacto que produce la acumulación en la atmósfera de dióxido de carbono de origen antropogénico -esencialmente procedente de la quema de combustibles fósiles- y ha desarrollado varias acciones con la finalidad de mitigarlo.

En el año 1992 -en la Cumbre de la Tierra de Ríose acordó la Convención Marco de las Naciones Unidas sobre el Cambio Climático (CMNUCC), con el objetivo de estabilizar "las concentraciones de gases de efecto invernadero en la atmósfera a un nivel que impida interferencias antropógenas peligrosas en el sistema climático" ${ }^{\text {. Cinco años }}$ después se formuló el Protocolo de Kyoto (1997), que fue el primer acuerdo -y el único hasta el momento- jurídicamente vinculante con objetivos cuantificados de limitación de emisiones de gases de efecto invernadero para los países más desarrollados. Actualmente, dado que la aplicación de este acuerdo finaliza en 2012, se están llevando a cabo negociaciones a nivel internacional, concretamente dentro del marco de la CMNUCC, para determinar un acuerdo que sirva de relevo al Protocolo de Kyoto. Las expectativas de la comunidad internacional estaban centradas en la conferencia de las partes de 2009, celebrada en Copenhague, pero no se alcanzaron los resultados esperados.

$8 \quad$ NACIONES UNIDAS, 1992. p. 4.
En la última conferencia, acontecida a finales de 2010 en Cancún, tampoco se pudieron definir las obligaciones y esfuerzos de los diferentes países y/o sectores de actividades, pero se reconoció como objetivo ambiental no superar en más de $2^{\circ} \mathrm{C}$ el aumento de la temperatura promedio global respecto a los niveles preindustriales ${ }^{9}$.

Fijar un límite numérico de aumento de la temperatura significa que se puede establecer la cantidad máxima de gases de efecto invernadero que se pueden emitir a nivel mundial. Esta cantidad viene determinada en el Cuarto Informe de Evaluación del Panel Intergubernamental de Expertos sobre Cambio Climático (IPCC). En este documento se especifica que para no aumentar la temperatura media mundial por encima de los $2^{\circ} \mathrm{C}$, es necesario que las emisiones mundiales de $\mathrm{CO} 2$ en el año 2050 sean entre un 50 y un $85 \%$ inferiores a las del año $2000^{10}$. Además, en aplicación del reparto del esfuerzo de mitigación acordado en la CMNUCC, en la que se reconocen las responsabilidades comunes pero diferenciadas entre países, se establece que los países desarrollados deben reducir entre el 80 y $95 \%$ de sus emisiones, mientras que los países en desarrollo no tienen un objetivo numérico, solo la indicación de alcanzar sustanciales desviaciones respecto a sus actuales tendencias de aumento ${ }^{11}$.

\footnotetext{
9 NACIONES UNIDAS, 2010. p. 2

10 BARKER, T. et al., 2007. p. 39.

11 IPCC, 2007. p. 776
} 
La lucha contra el cambio climático es el ejemplo más paradigmático de las restricciones que la sostenibilidad impone a la sociedad. En ese sentido, la aplicación del Protocolo de Kyoto supuso el inicio de un proceso de internalización del coste ambiental de uno de los elementos clave del sistema productivo industrial: las emisiones de gases de efecto invernadero.

\section{El sector de la edificación frente al reto de la sostenibilidad}

Todos los sectores productivos que componen nuestra sociedad dependen para su funcionamiento de la emisión de gases de efecto invernadero y, por lo tanto, todos deben -o deberán en un futuro próximo- reducir sus emisiones para poder seguir siendo competitivos, entendiendo por competitivos no solo la pervivencia de los sectores productivos, sino también la capacidad de proporcionar las utilidades socialmente demandadas.

El sector de la edificación no es una excepción y, a diferencia de otros sectores, debería dirigirse hacia la baja emisividad con la máxima urgencia, dado que la mayoría de los edificios que se construyen hoy seguirán en uso en el año 2050, momento en el que las emisiones mundiales deberían haberse reducido como mínimo a la mitad.

La edificación, además, representa uno de los sectores que más contribuye al cambio climático. Los datos disponibles indican que considerando únicamente las emisiones asociadas al uso de energía en los edificios -incluyendo las indirectas de la electricidad-, el sector fue el responsable del $33 \%$ de las emisiones de CO2 producidas en el año $2004^{12}$. Este porcentaje podría incrementarse significativamente si se considerasen también las emisiones asociadas a las otras fases de su ciclo de vida, sobre todo la correspondiente a la fabricación de los materiales de construcción de los edificios.

Las proyecciones futuras indican que las emisiones mundiales asociadas al uso de los edificios, que en el año 2004 fueron de 8,6 GtCO2, aumentarán, en el mejor de los escenarios, hasta las 11,4 GtCO2 para el año 2030 -o hasta las 15,6 GtCO2 en escenarios menos favorables ${ }^{13}-$. El análisis de estas estimaciones desagregadas por regiones y representado en la Figura 2, muestra que prácticamente todas las regiones aumentarán sus emisiones, y que solo en algún caso se estabilizarán. Incluso las regiones de los países desarrollados en los que la población está prácticamente estabilizada y se aplican medidas de reducción de las emisiones de

2 BARKER, T. et al., 2007. p. 54

13 BARKER, T. et al., 2007. p. 54. 


\section{FIGURA 2. EMISIONES DE C02 HISTÓRICAS (1971-2000) Y PROYECTADAS (2000-2030) ASOCIADAS AL USO DE LOS EDIFICIOS SEGÚN DIFERENTES ESCENARIOS.}
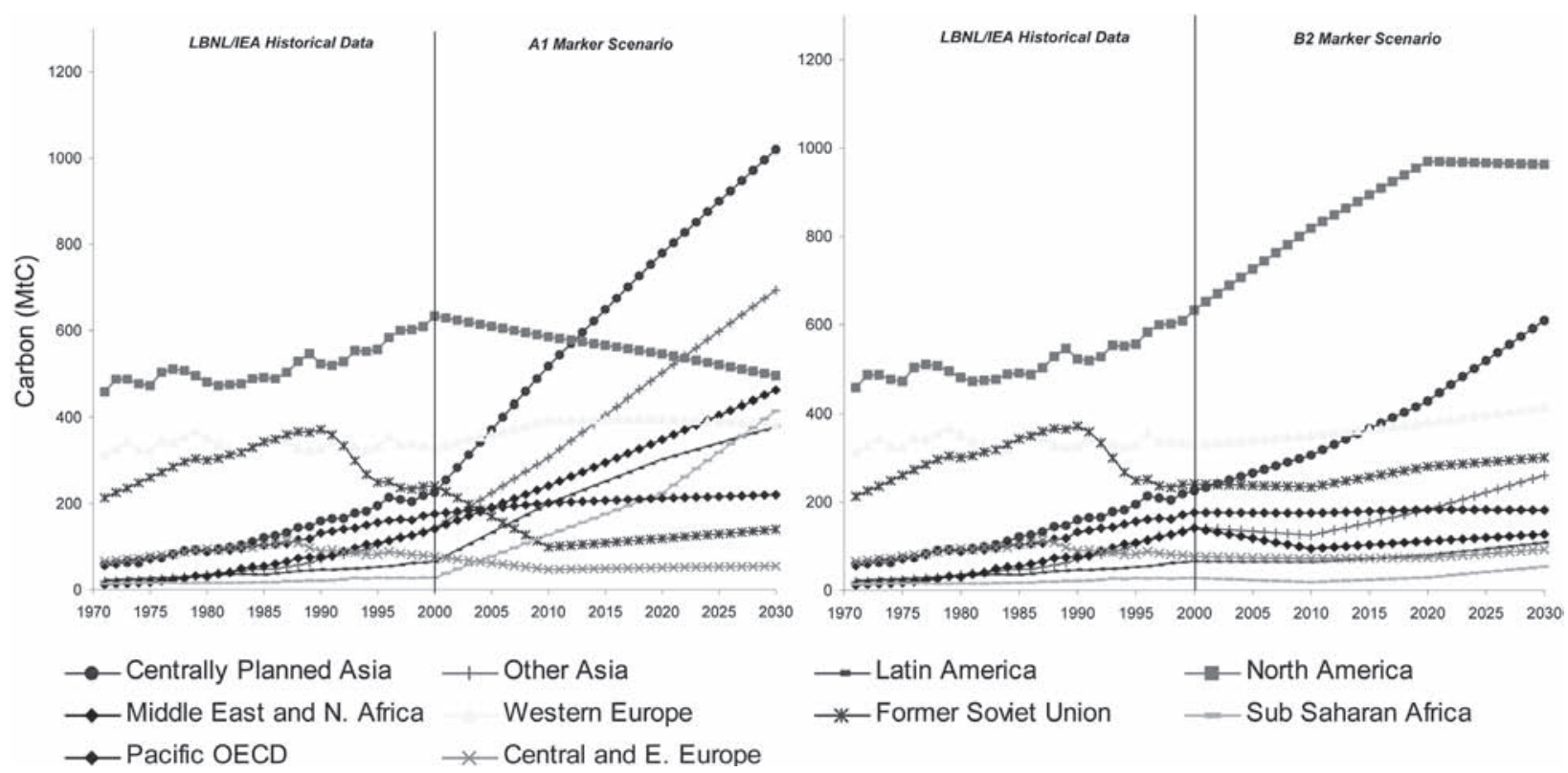

FUENTE: PRICE (PRICE, L. et al., 2006. p. 21)

$\mathrm{CO} 2$ en los edificios, no se observan reducciones sustanciales.

Estas circunstancias hacen necesaria una reflexión sobre los motivos por los que las acciones y estrategias de reducción de emisiones que se han aplicado y aplican actualmente sobre la edificación no son suficientes.

72 revista invi № 72 / Agosto 2011 / Volumen № 26: 65-93
La primera razón es que la sostenibilidad, incluyendo también la reducción de gases de efecto invernadero, se afronta generalmente desde la mejora de la eficiencia de los productos y servicios y/o de forma parcial:

- Las estrategias de aumento de la eficiencia de los productos están basadas en la reducción de

ARTíCULO: El futuro del hábitat: repensando la habitabilidad desde la sostenibilidad. El caso español / Joaquim Arcas-Abella, Anna Pagès-Ramon, Marina Casals-Tres 
los recursos y residuos vinculados a la producción y uso de un producto determinado. La reducción de los recursos se consigue por unidad de producto o servicio, pero no por el conjunto de productos o servicios que se han proyectado más eficientes. Más bien al contrario, el aumento de la eficiencia estimula un incremento del consumo, bien sea por un crecimiento del número de productos bien sea por el mayor uso que se da a los productos que han mejorado su eficiencia. Así, el ahorro inicial queda contrarrestado por un aumento del consumo a mediano plazo. Este fenómeno, denominado "efecto rebote" o "paradoja de Jevons"14, tiene su ejemplo más claro en la movilidad: aunque los automóviles son cada vez más eficientes por unidad de servicio, el impacto ambiental del sector del transporte crece año tras año como consecuencia del aumento del número de vehículos y del aumento del uso unitario.

En el caso de la edificación, son numerosas las acciones que intervienen sobre la eficiencia de los productos energéticos que se encuentran en el interior del edificio -etiquetado de electrodomésticos, iluminación, etc.- y sobre la eficiencia de los propios edificios -reducción de la demanda energética, certificación de edificios, etc.-, pero estas acciones no consideran la globalidad de edificios del sector y actualmente

14 POLIMENI J.M., et al., 2009 se aplican principalmente a nuevos edificios o productos. Por ejemplo en España, las emisiones asociadas al uso de los edificios destinados a vivienda en el periodo 1990-2005 se han mantenido prácticamente constantes por unidad de superficie $e^{15}$, mientras que el global de emisiones asociadas al uso del conjunto de este tipo de edificios ha aumentado en un 69\% durante el mismo periodo.

- Las estrategias de intervención parcial se basan en la reducción del uso de los recursos -o generación de residuos- de solo una parte del ciclo de vida de un producto o servicio. Normalmente las estrategias de este tipo comprueban el ahorro de recursos y residuos de la parte del proceso sobre el que intervienen, pero no tienen en cuenta el aumento de los impactos que se pueden derivar de la propia intervención en otras fases del ciclo de vida. De este modo, es imposible garantizar que la aplicación de la acción conlleve una reducción real del impacto ambiental.

En el caso de la edificación, existen estrategias que reducen el impacto de la fase de uso de los edificios - por ejemplo limitando la demanda térmica-, que al mismo tiempo pueden implicar un aumento del impacto en la fase de construcción, básicamente por usar materiales

15 PAGÈS, Anna. 2008. p.3 
y sistemas constructivos con un proceso de fabricación altamente energético.

La aplicación al sector de la edificación de metodologías de evaluación del impacto ambiental que consideren las diferentes fases de los edificios -como por ejemplo el análisis de ciclo de vida (ACV) - es relativamente reciente. A nivel internacional, la mayoría de estos estudios consideran el análisis de un elemento o sistema constructivo y pocos analizan el ciclo de vida de la globalidad del edificio. En España "el desarrollo de estudios de ACV (...) es claramente minoritario, tanto a nivel de edificios como a nivel de producto"16, lo que explica la inexistencia a nivel global del sector de estrategias de este tipo.

La segunda razón -desarrollada a partir del siguiente apartado- es que las estrategias autodenominadas sostenibles que actúan sobre el sector de la edificación se aplican generalmente sin tener en cuenta la finalidad de los edificios, es decir, sin considerar su utilidad. Estas estrategias normalmente consideran la cantidad y características de los recursos y residuos asociados a los productos obviando los posibles mecanismos de mejora que pueden aplicarse a la extracción de la utilidad de dichos productos por parte de los usuarios ${ }^{17}$. En el caso español, se han llevado a cabo experien-

16 ZABALZA, Ignacio, 2011. p. 380-381.

17 NøRGARD, Jørgen S., 2006. p.15-29.

74 revista invi № 72 / Agosto 2011 / Volumen № 26: 65-93 cias que avalan la capacidad de reducción de las emisiones de gases de efecto invernadero actuando únicamente sobre la utilidad de los edificios, por ejemplo modificando el programa funcional del edificio o ajustando los horarios ${ }^{18}$.

En general, cualquier intento de reducción del impacto ambiental de cualquier proceso debería, en primer lugar, plantearse desde la consideración de la utilidad que proporciona, puesto que no hay nada más insostenible que destinar recursos, aunque sean renovables, a un producto sin utilidad alguna o a una utilidad definida desde criterios consumistas.

En ese contexto, en el que las estrategias que se vienen aplicando son, como se ha expuesto, ineficaces para hacer frente a la presente crisis ambiental provocada por el metabolismo lineal propio de las sociedades minerales, se hace necesario rescatar el concepto fundamental de edificación sostenible e identificar los condicionantes que de él se derivan.

\section{Las dos claves de la edificación sostenible}

Tomando como referencia la definición de desarrollo sostenible que la Comisión Mundial sobre el Medio Ambiente y el Desarrollo (CMMAD) formuló en el año 1987 en el informe Nuestro futuro

18 MATA, É. et al., 2009. p.1334-1346.

ARTÍCULO: El futuro del hábitat: repensando la habitabilidad desde la sostenibilidad. El caso español / Joaquim Arcas-Abella, Anna Pagès-Ramon, Marina Casals-Tres 
común ${ }^{19}$-también conocido como informe Brundtland- es posible transponer sus exigencias y obtener una definición de edificación sostenible. Dicho informe enuncia que el desarrollo sostenible es "el desarrollo que satisface las necesidades de la generación presente sin comprometer la capacidad de las generaciones futuras para satisfacer sus propias necesidades". Los dos conceptos a considerar para llevar a cabo el paralelismo son satisfacer necesidades y no comprometer a las generaciones futuras.

Por un lado, el concepto no comprometer a las generaciones futuras debe ser leído como una condición al desarrollo: no alterar la cantidad de recursos disponibles ni degradar el medio por acumulación de residuos, es decir, cerrar los ciclos materiales de los procesos técnicos de satisfacción de las necesidades que forman parte de las actividades humanas. Cerrar los ciclos materiales, o dicho de otro modo, operar mediante un metabolismo circular, significa que el ritmo de extracción de recursos o generación de residuos asociado a las actividades humanas debe ser menor al de reposición de recursos o absorción de residuos por el medio. En consecuencia, el único modo de dirigir hacia la sostenibilidad nuestra sociedad industrializada consiste en actuar sobre cada uno de los ciclos materiales que actualmente deja abiertos, como, desde el año 2008, se empieza a realizar con el ciclo del carbono.

19 COMISIÓN MUNDIAL SOBRE EL MEDIO AMBIENTE Y EL DESARROLLO, 1988. p.67-70.
Por otro lado, satisfacer necesidades, sobre todo las esenciales -enunciadas en el informe Brundtland como alimento, ropa, abrigo y trabajo-, es el objetivo fundamental del desarrollo económico y social, y se alcanza mediante las utilidades que proveen -además de otros factores- el conjunto de bienes y servicios producidos por las actividades humanas, entre ellas las actividades que conforman el sector de la edificación.

Efectivamente, la articulación entre la dimensión humana de la necesidad y la dimensión física del bien destinado a satisfacerla, se realiza a través de la noción de utilidad: la utilidad es la prestación que emana de las características de un bien y que permite satisfacer una necesidad en un contexto cultural determinado. Y, en última instancia, es la utilidad la cualidad que se pierde en el proceso de utilización de los bienes que los convierte en residuos. En suma, se puede definir el consumo de utilidades como la operación de aprovechamiento de las condiciones que se obtienen del paso de productos -bienes organizados- a residuos, materiales desorganizados, con alta entropía, que ya no son capaces de satisfacer la necesidad para la cual fueron producidos ${ }^{20}$

La inserción de un eslabón entre necesidad y bien, propuesta ya en su momento por varios autores ${ }^{21}$,

20 CUCHÍ, A., 2010. p. 62

21 MAX-NEEF, M., et al., 1994. p. 50; DOYAL, L. y GOUGH, I. 1994. p. 200.

revista invi ํo 72 / Agosto 2011 / Volumen N ${ }^{0}$ 26: 65-93 
posibilita en primera instancia liberar el concepto de necesidad del relativismo cultural que habitualmente se le otorga para dotarlo de una dimensión universal, mientras que las utilidades devienen medios de satisfacción elegidos por una sociedad en un tiempo histórico concreto: "las necesidades de alimento y vivienda se aplican a todas las personas, pero existe una enorme variedad de cocinas y de formas de alojamiento diferentes, que pueden colmar cualquier especificación establecida en cuanto a la nutrición y la protección contra los elementos"22. Al fin, las prestaciones generadas por las actividades humanas se convierten en utilidades en tanto que tienen las características adecuadas para que los individuos sean física y culturalmente capaces de aprovecharlas.

En segundo lugar, la consideración de la noción de utilidad permite reescribir el objetivo del desarrollo humano, desvinculándolo de la producción de bienes para centrarlo en la generación del abanico de utilidades que cada sociedad demanda para satisfacer el conjunto de sus necesidades básicas. Utilidades que constituyen una lista abierta y que deben ser periódicamente identificadas y ordenadas en un ejercicio continuo de deliberación democrática, que constituya un verdadero proceso de auditoría social sobre su bondad para con las necesidades de la población actual y futura ${ }^{23}$.

22 GOUGH, I., 2007. p. 188.

23 TELLO, E., 2006. p. 72

76 revista invi № 72 / Agosto 2011 / Volumen № 26: 65-93
Desde esta perspectiva, es posible afirmar que el sector de la edificación, en el cumplimiento del papel esencial que le otorga la sociedad, a saber, resolver la necesidad universal de alojamiento de la población, debería garantizar la generación y el mantenimiento en el tiempo de su utilidad principal: la habitabilidad socialmente necesaria para acoger las actividades humanas. Del mismo modo que sin satisfacer necesidades no tiene sentido el desarrollo, sin habitabilidad no tiene sentido la edificación, por cuanto esta representa su primera función social.

Como resultado de la transposición de la definición de desarrollo sostenible, la edificación sostenible es la edificación que provee la habitabilidad socialmente demandada y opera cerrando los ciclos materiales de todas las actividades implicadas en este proceso (Figura 3). Cerrar los ciclos materiales y proveer habitabilidad son, por lo tanto, las dos claves de la sostenibilidad en el sector de la edificación. Y actualmente están siendo abordadas de forma desigual: la primera -el cierre de los ciclos materiales-, aunque presenta múltiples deficiencias, está extensamente reconocida y es objeto de un amplio conjunto de estrategias. La segunda -dotar de habitabilidad-, en cambio, no es percibida como elemento intrínseco de la edificación sostenible $y$, en muchos casos, ni siquiera supone ni

ARTÍCULO: El futuro del hábitat: repensando la habitabilidad desde la sostenibilidad. El caso español / Joaquim Arcas-Abella, Anna Pagès-Ramon, Marina Casals-Tres 
FIGURA 3. ESOUEMA DEL PROCESO DE TRANSPOSICIÓN DE LA DEFINICIÓN DE DESARROLLO SOSTENIBLE A LA EDIFICACIÓN

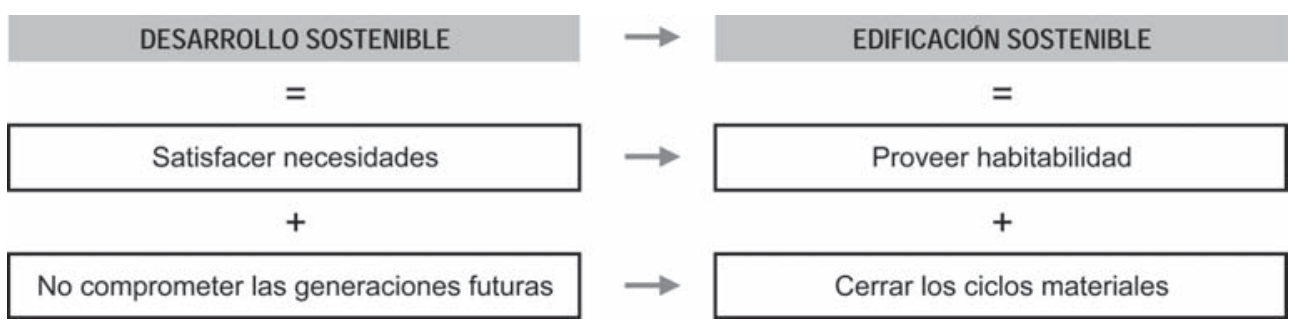

puede suponer el objetivo de la actividad edificatoria: la habitabilidad, entendida como las condiciones socialmente establecidas para satisfacer las necesidades de cobijo, presenta una escala que excede simultáneamente el ámbito de la concepción legislativa vigente y el campo de actuación físico y temporal de los agentes actuales.

\section{La necesaria redefinición de la habitabilidad. El caso español}

La consecución de la edificación sostenible, entendida como estrategia de presente y de futuro de configuración de un hábitat humano capaz de satisfacer las necesidades de cobijo de la población, supone una opción ahora ya innegociable para todas las sociedades del mundo, y conlleva asociada la tarea de análisis de los mecanismos de generación de habitabilidad, en aras de su redefinición orientada a partir de los condicionantes que emanan de la sostenibilidad.

Desde el presente artículo se propone una primera aproximación a dicho análisis y a dicha redefinición, a partir del estudio de las actuales circunstancias del Estado español, como ejemplo de país industrializado donde los mecanismos del sector de la edificación deberán ser profundamente alterados en pro de su viabilidad a largo plazo.

\section{MARCO Y OBJETIVOS DEL SECTOR DE LA EDIFICACIÓN}

La ausencia de una prolongación efectiva del derecho universal a una vivienda digna y adecuada, reconocido por la mayoría de las cartas magnas de los estados democráticos en forma de políticas 
públicas, ha permitido que durante décadas el sector de la edificación haya interpretado su encargo social de proporcionar habitabilidad bajo las reglas del desarrollo económico y, en consecuencia, haya centrado su actividad en producir edificios -principalmente viviendas- que, como bienes de consumo y por lo tanto de intercambio, puedan entrar en el mercado y en el juego del intercambio de mercancías. Un juego al que, como bien interpreta J.M. Naredo, ha estado y está actualmente sujeto el sector de la edificación cuando enuncia que la mayor parte de las inversiones del último auge económico han estado "más orientadas a obtener plusvalías que a producir bienes y servicios asociados a una mejor calidad de vida de la población"24.

El caso español supone un ejemplo paradigmático de país aspirante a extender el Estado del bienestar a todos sus conciudadanos donde, bajo la dinámica de crecimiento desenfrenado del sector de la edificación que lo ha caracterizado estos últimos años -motivada principalmente por razones de índole financiera y legitimada socialmente por los elevados beneficios económicos de particulares y por el incremento del Producto Interior Bruto que aportaban al país ${ }^{25}$-, la producción de viviendas ha alcanzado cotas sin precedentes -más de 5 millones en los últimos 10 años-, aunque con una producción que poco o nada tiene que ver con la

24 NAREDO, José Manuel, 2009. p. 118.

25 CORTÉS, Luís, 2009. p. 42.

78 revista invi № 72 / Agosto 2011 / Volumen N ${ }^{0}$ 26: 65-93 demanda social de alojamiento y con las variables demográficas: su actividad no ha cubierto la demanda de la habitabilidad socialmente necesaria.

En primer lugar porque la sobreproducción de viviendas no ha cubierto la demanda social de estas, puesto que junto al alza de los precios experimentada en la última década se ha dado una menor presencia de vivienda social ${ }^{26}$. En este sentido, se ha dado la paradoja que, simultáneamente a la fluidez del mercado de la vivienda, se ha desarrollado una crisis de accesibilidad entre los sectores sociales más débiles, motivada por varios factores: la consideración de la vivienda como bien de intercambio y sujeto de especulación económica, la ausencia de un parque de viviendas libres y públicas en alquiler con precios moderados, la existencia de un gran número de viviendas vacías, secundarias o turísticas, que no pueden ser utilizadas como principales, y la concentración de la oferta inmobiliaria en los segmentos residenciales más rentables ${ }^{27}$.

En segundo lugar porque los modelos de vivienda que ha ofertado el sector de la edificación español han sido cada vez menos variados, ocasionando graves dificultades de adaptación a un gran número de colectivos: jóvenes, inmigrantes, personas con movilidad residencial, parejas en trámites de separación, etc. Las causas de la actual rigidez de la oferta de vivienda se encuentran vinculadas a

26 NAREDO, José Manuel, 2004. p. 148.

27 CORTÉS, Luís, 2005. p. 81-100. 
factores culturales que han provocado un proceso histórico de selección de modelos habitables y, en los últimos años, a factores económicos, en cuanto la homogenización de la vivienda facilita su puesta en el mercado ${ }^{28}$.

Alcanzar la sostenibilidad en el sector de la edificación en España, y en todos los países que actualmente presentan la dinámica descrita de generación del hábitat humano, requiere redefinir esta forma de operar y, en consecuencia, precisa analizar y comprender el concepto de habitabilidad vigente para redefinirlo en función de los condicionantes de la sostenibilidad.

\section{SOBRE EL CONCEPTO DE HABITABILIDAD ACTUAL}

La definición del término habitabilidad -común en los países de habla hispana- dicta: "Cualidad de habitable, y en particular la que, con arreglo a determinadas normas legales, tiene un local o una vivienda" ${ }^{29}$. Dicha definición contiene, como veremos, los dos elementos principales que fundamentan el concepto de habitabilidad actual, a saber, que es un concepto normativo y que se reconoce principalmente sobre la vivienda. En el caso español, dicho reconocimiento se encuentra implícitamente recogido en la Constitución española

28 NAREDO, José Manuel y CUCHÍ, Albert, 2007. p. 90.

29 REAL ACADEMIA ESPAÑOLA, 2001. cuando se enuncia el derecho a "disfrutar de una vivienda digna y adecuada"30.

En este sentido, la habitabilidad se encuentra definida a partir del conjunto de legislaciones destinadas a hacer efectivo dicho derecho y, por tanto, deviene una propiedad que emana de las condiciones de un espacio que cumple una serie de parámetros mínimos dictados por la legislación correspondiente. De este modo, se considera un espacio habitable o no habitable, en función de si reúne la serie de requerimientos establecidos por la normativa de habitabilidad vigente en cada ámbito geográfico.

En el complejo panorama legislativo español existen divergencias tanto en los mecanismos de regulación de la habitabilidad, como en las exigencias que la fundamentan. Actualmente la regulación de la habitabilidad se establece desde dos escalas administrativas distintas: en algunos casos, mediante normas de habitabilidad de rango autonómico que disponen de Cédula de habitabilidad como documento de control; en otros casos, a través de ordenanzas de habitabilidad de rango municipal, en aquellas autonomías que han traspasado las competencias a los ayuntamientos. En cierto modo, las normativas técnicas de edificación -amparadas hoy por el Código Técnico de la Edificación ${ }^{31}$ - determinan otro tipo de condiciones adicionales para

30 ESPAÑA. Congreso de los Diputados, 1978.

31 ESPAÑA, 2006; ESPAÑA, 2010. 
la nueva edificación o el resultado de una gran rehabilitación.

En relación con las condiciones mínimas exigidas por cada administración, las divergencias se manifiestan en la propia organización del documento y en los parámetros funcionales y constructivos, estableciendo requisitos dimensionales, de equipamientos, de suministros o de acabados, a menudo poco o nada coincidentes.

Ahora bien, a pesar de las diferencias circunstanciales existentes entre las distintas regulaciones de la habitabilidad, todas ellas presentan unos rasgos estructurales idénticos, heredados de un antecesor común, la Orden de 29 de febrero de 1944, por la que se establecen las condiciones higiénicas minimas que han de reunir las viviendas ${ }^{32}$. Dicha orden, como normativa pionera en el Estado español, estableció las características fundamentales sobre las que se ha erigido toda la legislación posterior y que, en buena medida, todavía hoy articulan el concepto de habitabilidad de nuestra sociedad. Características -descritas en los cuatro subapartados siguientes- que deben ser remarcadas y analizadas para verificar su papel en la definición de una habitabilidad sostenible.

32 ESPAÑA, 1944. p. 1833-1834.

80 revista invi № 72 / Agosto 2011 / Volumen № 26: 65-93

\section{A. UnA HABITABILIDAD EXPRESADA SOBRE CONDICIONES MATERIALES}

En primer lugar, en todas las normativas la habitabilidad se expresa directamente sobre condiciones materiales concretas, es decir, desde la definición de requerimientos sobre parámetros constructivos, desde la exigencia de unos bienes determinados en forma de espacios -salón, dormitorio, cocina, baño, etc.-, de equipamiento -fregadero, fogones, lavabo, inodoro, ducha-, o de instalaciones y suministros -agua caliente y fría, energía eléctrica, telecomunicaciones-.

De este modo, la habitabilidad se enuncia sin una ordenación explícita de las necesidades básicas a cubrir, puesto que no se aporta ninguna definición de habitabilidad que plantee las funciones o actividades humanas a las que se debe dar cobijo ni la forma en que deben ser satisfechas. Excepto en algún caso, en lugar de reconocer necesidades como alimentarse, vestirse o higienizarse, se especifican unos bienes materiales como son el comedor, la lavadora o la bañera.

El resultado de no trabajar desde las necesidades puede llevar a falta de condiciones para el adecuado desarrollo de la vida de las personas, pues un 
modelo de habitabilidad inconsciente de la amplia variedad de demandas que debe cubrir, difícilmente puede proporcionarles una respuesta adecuada. Dicha inadecuación puede conducir a un consumo inapropiado y, por lo tanto, ineficiente de los recursos, al ofrecer productos que pueden no corresponder a la utilidad demandada.

\section{B. UNA HABITABILIDAD IDENTIFICADA CON UN LIMITADO ABANICO DE MODELOS DE VIVIENDA}

En segundo lugar, el modelo de habitabilidad implícito en todas las normativas españolas se encuentra identificado con un limitado abanico de modelos de vivienda, destinados no solo a albergar a un perfil restringido de habitantes - basado en la familia nuclear-, sino a responder a un intervalo concreto de convivencia -los cónyuges con hijos dependientes-. Esta limitada gama programática, como se ha expuesto, viene incentivada históricamente por factores culturales que han potenciado la adopción de la vivienda burguesa como modelo de referencia. Y recientemente, por factores económicos, como la conversión de la vivienda en un bien de mercado sobre el cual prevalece su valor de cambio por encima de su valor de uso, que con tal de dinamizar su intercambio han impulsado su homogeneización.

Como consecuencia de la rigidez de la oferta de vivienda, y por lo tanto del modelo de habitabilidad, se dan situaciones en las que no se responde adecuadamente a la diversidad de modos de habitar actuales ni a su evolución en el tiempo, perdiendo de este modo la capacidad de cumplir con su papel social de cobijar al conjunto de la sociedad y contraviniendo el mandato constitucional de disponer de una vivienda digna y adecuada. Al mismo tiempo este desfase entre oferta y demanda supone un desaprovechamiento de los recursos invertidos en aquellos casos en que las prestaciones de la vivienda exceden las necesidades de sus habitantes.

\section{UNA HABITABILIDAD RECLUIDA A LA ESCALA DOMÉSTICA}

En tercer lugar, las normativas actuales de habitabilidad se expresan únicamente sobre la vivienda, adoptando la escala doméstica como marco de aplicación de las exigencias de habitabilidad. Esta determinación se desarrolla sin tener en cuenta que las distintas funciones domesticas están supeditadas a factores que escapan de la escala de la vivienda y del actual marco normativo de la habitabilidad, como son -por ejemplo- la tipología edificatoria definida por la trama urbana, o el tipo de infraestructuras y flujos que sirven a un domicilio, entre otros. Asimismo, las propias actividades que acoge la casa no pueden entenderse desvinculadas de otras que suceden fuera de sus límites físicos y legales, y que inexorablemente forman parte del proceso de satisfacción de necesidades que culmina dentro del espacio doméstico. Claro está que el acto de alimentarse, reconocido implícitamente en la exigencia normativa de disponer de un espacio equipado llamado cocina, se despliega a través de 
una sucesión de acciones que empiezan, en la mayoría de los casos, en el mercado, servicio público situado en la calle y, por lo tanto, fuera del alcance de la normativa de habitabilidad, aunque su accesibilidad sea un factor determinante en los costes ambientales y económicos de satisfacer aquella necesidad.

Todos estos aspectos excluidos de la actual normativa de la habitabilidad, que son decisivos en su conformación, se abordan en el panorama legislativo español actual desde distintos ámbitos, desde administraciones diversas y con escalas espaciales y geográficas muy diferentes. A grandes rasgos se pueden identificar, por lo bajo, tres campos legales que intervienen en la habitabilidad sin ser conscientes de ello ni plantear estrategias de coordinación: normativas técnicas, planeamiento urbano y planeamiento territorial.

Esta disgregación actual es el resultado de la carencia de una definición global del concepto de habitabilidad y, en algunos casos, de la ignorancia del papel que esa normativa juega en la modelación de la habitabilidad, y conlleva graves disfunciones al no incorporar y coordinar bajo un mismo enfoque todos los elementos que hoy en día conforman la habitabilidad y generan la demanda de recursos y residuos para proveerla.

En consecuencia, el enfoque normativo segregado actual deviene en factor negativo en el camino hacia la sostenibilidad, puesto que al actuar de forma parcial sobre los procesos, esto es interviniendo por escalas en búsqueda de reducciones en el consumo de recursos y generación de residuos, puede suponer mejoras puntuales aparentes que conllevan, en realidad, aumentos globales del impacto ambiental.

\section{UNA HABITABILIDAD PLANTEAdA SIN CONSIDERAR LOS RECURSOS Y RESIDUOS IMPLICADOS}

En cuarto lugar, y exceptuando algún caso notorio como es la eficiencia energética recogida en el Código Técnico de la Edificación, el concepto actual de habitabilidad se define con independencia de los recursos y residuos implicados en su consecución y mantenimiento en el tiempo, aunque determina su ordenación y gestión. De esta manera, el constante aumento de los estándares normativos de confort, funcionales o constructivos de la habitabilidad ha conllevado un incremento paralelo en el consumo de recursos y generación de residuos que no ha sido evaluado.

Asimismo, frente al reto sostenible aplicado a la habitabilidad y a su sujeto -la vivienda-, las legislaciones recientemente aprobadas actuan exclusivamente mediante medidas de aumento de eficiencia en la fase de uso de los edificios. El Código Técnico de Edificación incluye únicamente la limitación de la demanda energética, el rendimiento de las instalaciones térmicas y la eficiencia energética de las instalaciones de iluminación. Dichas estrategias no recogen la totalidad de flujos materiales

ARTíCULO: El futuro del hábitat: repensando la habitabilidad desde la sostenibilidad. El caso español / Joaquim Arcas-Abella, Anna Pagès-Ramon, Marina Casals-Tres 
que intervienen en mantener las condiciones de habitabilidad de una vivienda -como son el agua, el gas natural o gases licuados del petroleo, y los residuos sólidos urbanos- ni los recursos comprometidos en las fases de construcción que, en la mayoria de los casos, pueden suponer entre el 30 y el $50 \%$ de las emisiones globales del edificio ${ }^{33}$.

\section{ConseCuencias de la articulación Actual de la HABITABILIDAD}

El resultado de la articulación de la habitabilidad actual a partir de los cuatro principios aquí expuestos produce una perenne incapacidad de extender a todos los colectivos esa utilidad socialmente demandada, no solo ya de disponer de una vivienda digna y adecuada -tal y como exige la Constitución española-, sino también, y esta es una de la cuestiones clave, de garantizar una habitabilidad que se desarrolle a la escala de los procesos de satisfacción de necesidades, es decir, a la escala urbana.

Análogamente, el concepto de habitabilidad actual se muestra incapaz de asimilar la exigencia de la sostenibilidad, puesto que se encuentra restringido en un campo semántico desde el cual no es posible actuar de forma coherente con la magnitud y complejidad del cambio que conlleva la reducción de la emisividad del modelo actual. Principalmente porque no contempla las dinámicas materiales

33 CUCHÍ, A., 2010. p. 49. que lo sustentan ni dispone de la escala adecuada para contemplarlas.

Ante esta situación, deviene imprescindible una redefinición del concepto de habitabilidad que lo capacite para afrontar las problemáticas sociales y medioambientales descritas de forma global. Una redefinición capaz de comprender de forma integral el patrón de estructuras y acciones que permiten la satisfacción de las necesidades de cobijo socialmente demandadas a partir de los flujos materiales intercambiados con el entorno, y que permita el desarrollo de estrategias de transformación de dicho modelo.

\section{PRINCIPIOS PARA LA REDEFINICIÓN DEL CONCEPTO DE HABITABILIDAD}

Esta redefinición debe partir de la consideración de la habitabilidad como respuesta a la demanda social de accesibilidad y disponibilidad a las utilidades precisas para satisfacer un conjunto de necesidades socialmente determinadas y reconocidas, y hacerlo desde la consideración del consumo de recursos implicados en obtenerla y desde las restricciones sociales a la generación de residuos que ello ocasione. En este sentido, una habitabilidad interpretable a partir del uso del conjunto del patrimonio existente en el tiempo, y no como una propiedad puntual de una edificación recién construida. 


\section{FIGURA 4. ESQUEMA DE LOS PRINCIPIOS DE LA REDEFINICIÓN DE LA HABITABILIDAD CONTRAPUESTOS A LA HABITABILIDAD ACTUAL}

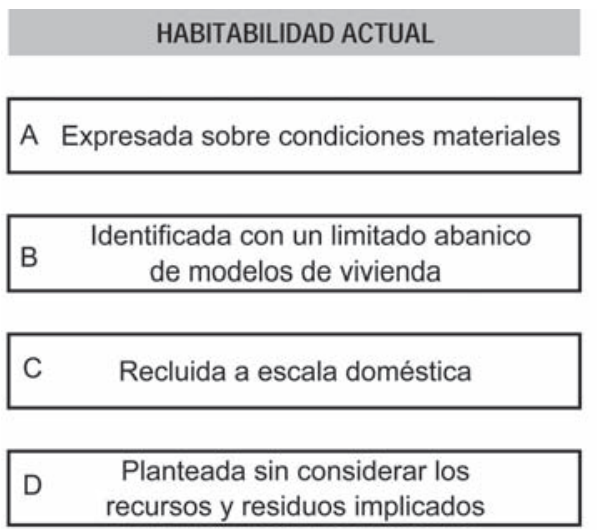

En consecuencia, la definición de la habitabilidad debe basarse en principios necesariamente contrapuestos a los de la concepción actual (Figura 4).

\section{A. Una habitabilidad ENUNCIADA DESDE LA PERSONA Y SUS NECESIDADES}

En primer lugar, la habitabilidad debería vincularse a la persona y sus necesidades, y por lo tanto, reconocer que existe una amplia variedad de situaciones -en función de la edad, sexo, condición social y cultural, renta, entorno, etc.- que exigen respuestas concretas derivadas de esta especificidad. Asociar la habitabilidad a la satisfacción de necesidades conlleva articularla desde el consumo y no, como hasta ahora, desde la producción de es-

84 revista invi № 72 / Agosto 2011 / Volumen N ${ }^{0} 26: 65-93$

\section{REDEFINICION DE LA HABITABILIDAD}

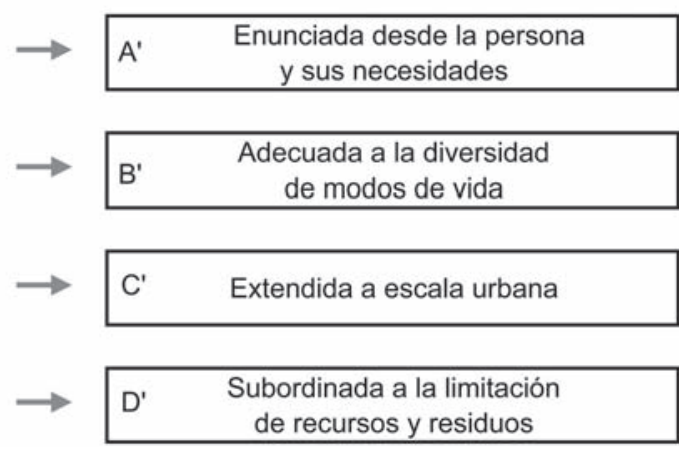

pacios definidos por soluciones materiales. De este modo, una habitabilidad interpretable principalmente a partir de las demandas sociales de cobijo debe articularse desde el consumo, concretamente desde el consumo de las utilidades precisas para la satisfacción de necesidades, y no, como hasta ahora, ser el resultado de la producción de unos espacios.

De este modo la habitabilidad, en lugar de expresarse sobre soluciones materiales concretas, como es por ejemplo la exigencia de instalar una lavadora, debe iniciarse con el reconocimiento de las necesidades a cubrir, en este caso disponer de ropa limpia para abrigarse, y garantizar su satisfacción mediante alguna de las prestaciones y servicios

ARTÍCULO: El futuro del hábitat: repensando la habitabilidad desde la sostenibilidad. El caso español / Joaquim Arcas-Abella, Anna Pagès-Ramon, Marina Casals-Tres 
socialmente admitidas, esto es tener una lavadora en el domicilio, usar una lavadora compartida con los vecinos o contratar un servicio de lavandería. En consecuencia, una habitabilidad que no fija condiciones materiales preconcebidas sino que se enuncia de forma exigencial y admite múltiples estrategias de gestión de las utilidades en función de cada individuo y sus demandas concretas, desde disponer de ciertas condiciones materiales -la lavadora doméstica- hasta recibir un servicio -lavado y planchado de la ropa-.

En esta dirección contribuyen líneas argumentales auspiciadas tanto por el desaparecido Ministerio de la Vivienda -ahora integrado en el Ministerio de Fomento- como la elaborada por el grupo de investigación Habitar -Universitat Politècnica de Catalunya-, que bajo el proyecto teórico Rehabitar defiende una visión de la arquitectura que parte de la persona. La propuesta se basa en intervenir en el parque existente desde la consideración de las acciones y procesos desencadenados por sus habitantes: "Son las personas quienes tienen la capacidad de transformar un espacio y, por tanto, son esenciales para rehabitarlo. (...). Mientras rehabilitar hace referencia al objeto, rehabitar hace referencia a la acción, al sujeto que la produce" 34 .

El resultado de reconocer las necesidades de las personas implica considerar una habitabilidad di-

34 MONTEYS, X. et al., 2010. p. 15. versificada que proporcione las condiciones adecuadas a la gran variedad de demandas sociales presentes en nuestra sociedad y, por lo tanto, que ajuste los recursos invertidos a cada circunstancia.

\section{B. UNA HABITABILIDAD ADECUADA A LA DIVERSIDAD DE MODOS DE VIDA}

En segundo lugar, la habitabilidad debería ser diversificada y adaptable, y proporcionar respuestas adecuadas a la variedad de modos de vida y estructuras de convivencia presentes en la actualidad y a su evolución en el tiempo.

En contraposición a la actual rigidez de la oferta de vivienda del modelo residencial español, se debería reconocer el amplio abanico de expresiones de la habitabilidad socialmente aceptadas hoy en día. De manera que, en lugar de reproducir únicamente modelos de vivienda fundamentados en la familia nuclear, se puedan proporcionar las utilidades demandadas mediante soluciones habitacionales ajustadas a las demandas específicas de colectivos cada vez más presentes en nuestra sociedad, como son solitarios, inmigrantes, parejas sin hijos, hogares monoparentales, grupos cohabitantes, familias extensas, familias múltiples, etc.

La diversificación de las configuraciones de la habitabilidad debería estar orientada a ofrecer condiciones materiales ajustadas a las necesidades 
concretas de los distintos modos de vida urbanos acordando, de este modo, oferta y demanda, y optimizando los recursos invertidos en la consecución y mantenimiento de la habitabilidad.

Al mismo tiempo, la lectura precisa de las utilidades que supone la pluralidad de la habitabilidad debería permitir reconocerlas y recuperarlas en el parque de edificios existente con la máxima precisión, aportando una mayor eficiencia en el uso del patrimonio y optimizando la habitabilidad que contiene.

\section{UNA HABITABILIDAD EXTENDIDA A ESCALA URBANA}

En tercer lugar, la habitabilidad debe plantearse a escala urbana y englobar bajo un mismo enfoque el conjunto de utilidades que permiten la satisfacción de necesidades socialmente reconocidas, considerando transversalmente desde el ámbito doméstico hasta el espacio urbano.

La habitabilidad debe superar su actual marco de aplicación -la vivienda- y abarcar la escala urbana para poder coordinar la totalidad de elementos involucrados en la satisfacción de necesidades. Por un lado, porque tanto la vivienda como las actividades que acoge están condicionadas por factores urbanos. Sirva como ejemplo el mencionado caso de la alimentación: a saber, las funciones de cocinar y comer exigen la adquisición de alimentos en espacios situados fuera de la casa. Por el otro, porque el número de necesidades a las que debe dar respuesta la habitabilidad, en lugar de ceñirse a las domésticas como hasta ahora, debe expandirse al conjunto real de necesidades de las personas, es decir, a las propias de la vida urbana. Igual que no se puede admitir una configuración de la habitabilidad sin condiciones para el descanso, el abrigo o la higiene, tampoco es aceptable hoy en día que los ciudadanos no dispongan de acceso a la educación, la sanidad, la cultura, el deporte o la compra de bienes diversos, es decir, a los servicios esenciales para una ciudadanía digna que ofrece la ciudad.

El cambio de escala de la habitabilidad -de la doméstica a la urbana- conlleva la consideración de condiciones en la satisfacción de necesidades hasta ahora obviadas. En primer lugar, la habitabilidad incorpora, junto a las condiciones materiales que conforman el lugar de satisfacción de una necesidad, las condiciones de accesibilidad hasta este lugar. Claro está que un servicio no podrá considerarse disponible si el tiempo o coste de acceso hasta él excede las posibilidades de la persona que lo precisa. En segundo lugar, la habitabilidad a escala de la ciudad permite entender que la satisfacción de necesidades se produce en distintos grados de privacidad, mediante el uso de ciertos bienes compartidos con grupos más o menos numerosos que ponen en común unos recursos. En este sentido, cada una de las demandas que conforman la habitabilidad del individuo dispone de unas condiciones de privacidad socialmente aceptables, así para el descanso o la higiene se procura la máxima in-

ARTíCULO: El futuro del hábitat: repensando la habitabilidad desde la sostenibilidad. El caso español / Joaquim Arcas-Abella, Anna Pagès-Ramon, Marina Casals-Tres 
timidad, en cambio, para la alimentación o el ocio, se acepta una menor privacidad.

Este enfoque global de la habitabilidad urbana, que plantea el consumo total de utilidades desde la persona, permite optimizar los mecanismos de satisfacción de necesidades y, simultáneamente, maximizar la capacidad de intervención sobre las dinámicas materiales del metabolismo urbano, ofreciendo la posibilidad de plantear, por ejemplo, estrategias de reducción del total de emisiones de gases de efecto invernadero asociados a la generación y mantenimiento de la habitabilidad.

En este sentido, dicho enfoque puede devenir un eficaz instrumento de estructuración de la ciudad, tanto en relación con nuevos crecimientos como en la rehabilitación de la ciudad existente.

\section{UNA HABITABILIDAD SUBORDINADA A LA LIMITACIÓN DE RECURSOS Y RESIDUOS}

En cuarto lugar, la habitabilidad debería estar supeditada al cierre de los ciclos materiales, y en consecuencia, vinculada a los recursos precisos para proveerla y mantenerla en el tiempo, y limitada por la capacidad de emisión de residuos al medio que será progresivamente restringida.

La habitabilidad, como principal utilidad social que procura el sector de la edificación, requiere el uso de cierta cantidad de recursos y conlleva la generación de un determinado volumen de emisiones de gases de efecto invernadero, tanto para su consecución - su construcción- como para su mantenimiento a lo largo del tiempo. La relación entre ambos términos -habitabilidad vs. recursos y residuos- se expresa en las mencionadas condiciones materiales, condiciones de accesibilidad y condiciones de privacidad, y es bidireccional: la habitabilidad se conforma en función de la disponibilidad de recursos de cada momento histórico y, al mismo tiempo, condiciona el impacto ambiental del modelo en el futuro.

La consideración de esta relación recíproca pone de manifiesto dos cuestiones cruciales: la generación de habitabilidad va a estar progresivamente limitada por las restricciones sociales a la emisión de gases de efecto invernadero; y la disponibilidad de habitabilidad en el futuro estará vinculada a la eficiencia en el empleo de recursos y generación de residuos del modelo del presente, pues la edificación actual, a diferencia de otros productos más perecederos, permanecerá en uso durante las próximas décadas.

En el caso español se han llevado a cabo durante los últimos años numerosas iniciativas públicas y privadas de incorporación de soluciones de bajo impacto ambiental a proyectos de vivienda. Sin embargo, aunque cualitativamente valiosos, son cuantitativamente poco relevantes frente a la dimensión de los retos globales que presenta el parque edificado, constituido actualmente por más de 
25 millones de viviendas ${ }^{35}$. Considerando el consumo energético, cabe destacar que el 58\% de las viviendas fueron construidas sin norma térmica ${ }^{36}$ y que alrededor del 38\% fueron construidas con alguna normativa térmica pero sin las últimas exigencias de eficiencia energética del Código Técnico de la Edificación.

En conclusión, desde la responsabilidad de procurar la habitabilidad socialmente necesaria, cabe señalar la importancia de dirigir al conjunto del sector de la edificación hacia la baja emisividad, mediante una concepción de la habitabilidad que proporcione las directrices que permitan actuar sobre el modelo actual y subordinarlo a los límites ambientalmente asumibles.

\section{IDONEIDAD DE LA REDEFINICIÓN DE LA HABITABILIDAD}

En suma, se propone una habitabilidad basada en principios contrapuestos a la concepción normativa vigente, a saber, una habitabilidad enunciada más desde la persona y sus necesidades y demandas, más que solo desde las propiedades de unos espacios; una habitabilidad planteada a escala urbana que contemple globalmente las acciones y los procesos materiales que permiten la satisfacción de necesidades; una habitabilidad subordinada a

35 INSTITUTO Nacional de Estadística, 2005; MINISTERIO DE FO-

36

88 revista invi № 72 / Agosto 2011 / Volumen № 26: 65-93 la disponibilidad de recursos y a las limitaciones a la emisión de residuos.

La redefinición de la habitabilidad desde la consideración de los principios expuestos la capacitaría conceptualmente para hacer frente a los principales retos que plantea el paradigma de la sostenibilidad en la edificación, a saber, proporcionar un alojamiento digno para todas las personas, ajustado a sus demandas y, al mismo tiempo, limitado en el uso de los recursos y la generación de los residuos.

\section{Conclusiones}

Alcanzar y mantener en el futuro las condiciones habitacionales exigibles, desde el punto de vista del derecho universal, a una vivienda digna y adecuada conlleva redefinir los mecanismos actuales de generación del hábitat hacia la edificación sostenible, entendida como aquella que provee la habitabilidad socialmente necesaria para todos en un marco limitado de consumo de recursos y generación de residuos.

Esta exigencia nace, como se ha expuesto, de la necesidad de dar respuesta al inaplazable proceso de mitigación del impacto ambiental de las activi- 
dades humanas sobre el medio que nos sustenta $y$, en especial, de limitación de las emisiones de gases de efecto invernadero dadas sus inminentes consecuencias sobre el clima.

La magnitud del reto que plantea la sostenibilidad al sector de la edificación, junto con la urgencia con que debe ser abordado, conduce a formular dos estrategias generales vinculadas al nivel de déficit habitacional de cada ámbito geográfico.

Para las regiones con parques edificados que se encuentren lejos de satisfacer cuantitativa y cualitativamente las demandas de habitabilidad exigibles para con unas condiciones de vida socialmente aceptables, la condición de sostenibilidad se expresará en la necesidad de edificar nuevos asentamientos capaces de corresponder a las progresivas restricciones de impacto ambiental. Esto es, los resultados de la actividad de construcción de nuevas viviendas, nuevos barrios y nuevas ciudades deberán alcanzar el objetivo de no rebasar los límites fijados de emisión de gases de efecto invernadero y otros componentes contaminantes, teniendo en cuenta todo el ciclo de vida de la edificación, es decir, desde la fase ejecutoria y la de utilización, hasta la de desintegración.

En este proceso, la consideración de la segunda clave de la edificación sostenible presentada en este artículo, proporcionar habitabilidad, debería ser tenida en cuenta con el fin de asegurar un empleo óptimo de los recursos disponibles. Y en este sentido, cobra especial importancia la necesidad de redefinirla desde los principios expuestos: una habitabilidad que emana de las demandas de las personas y que abarca la escala urbana, pues es la escala en la que se desarrollan las necesidades de cobijo de la población.

Para las regiones que, en cambio, dispongan en la actualidad de un patrimonio construido con un nivel de habitabilidad medio objetivamente suficiente para albergar al conjunto de su población -como el expuesto caso español-, las estrategias de sostenibilidad deberán considerar, principal y justamente, la rehabilitación de dicho patrimonio residencial como campo de actuación decisivo de la edificación sostenible.

En primer lugar porque, a diferencia del caso anterior, el único modo de reducir el impacto ambiental actual del conjunto del sector no pasa por construir nueva edificación sino por intervenir en el parque construido, optimizando el uso de recursos y la generación de residuos por unidad de habitabilidad proporcionada. Pocas posibilidades presenta el sector de la edificación si, junto a la construcción de nuevos edificios sostenibles, no rehabilita la fuente principal de emisiones: el patrimonio existente.

En segundo lugar, porque el patrimonio construido es la principal fuente de utilidades actual de estas regiones $y$, en consecuencia, cualquier transformación del modelo de habitabilidad debe 
ser abordada desde la rehabilitación de su parque existente. Poca repercusión tendría para la mayoría de la población el planteamiento de nuevas estrategias de satisfacción de necesidades aplicadas a la nueva construcción, pues suponen un porcentaje despreciable del fondo de habitabilidad de cada una de estas áreas geográficas.

El resultado de las exigencias de sostenibilidad en estas regiones implica cambiar la dinámica del sector de la edificación, basada en la nueva construcción, para redirigirla hacia la rehabilitación. Una rehabilitación enfocada a mantener y optimizar la habitabilidad disponible en el parque existente $y$, en consecuencia, que actúe proporcionando las utilidades justas y adecuadas a las necesidades de todas las personas, que tome la escala urbana como campo de intervención, y que cierre los ciclos materiales de todos los procesos técnicos implicados en su consecución.

\section{Referencias bibliográficas}

BARKER, Terry. [et al.]. Resumen técnico. En: CAMBIO climático 2007: Mitigación. Contribución del Grupo de Trabajo III al Cuarto Informe de Evaluación del Panel Intergubernamental de Expertos sobre Cambio Climático. Cambridge, Cambridge University Press. 96 p. [En línea]. IPCC - Intergovernmental Panel on Climate Change. 2007. Disponible en: http://www.ipcc.ch/pdf/assessmentreport/ar4/wg3/ar4-wg3-ts-sp.pdf

COMISIÓN Mundial Sobre el Medio Ambiente y el Desarrollo. Nuestro futuro común. Madrid, España, Alianza Editorial. 1988. 460 p. ISBN 8420695742

CORTÉS, Luis y NAVARRETE, Jimena. Reflexiones en torno al sistema residencial y el derecho a la vivienda en nuestra sociedad. Sociedad y Utopía. Revista de Ciencias Sociales. (33): 37-63, junio 2009. ISSN 1133-6706

CORTÉS, Luis. La crisis de la vivienda. Documentación Social. (138): 81-100, julio 2005. ISSN 0417-8106.

CUCHÍ, Albert; WADEL, Gerardo y RIVAS, Paula. Cambio global España, 2020/50: Sector edificación: la imprescindible reconversión del sector frente al reto de la sostenibilidad. Madrid, España, Fundación General de la Universidad Complutense. 2010. 248 p. ISBN 9788461404575.

DOYAL, Len y GOUGH, Ian. Teoría de las necesidades humanas. Barcelona, España, Icaria. 1994. 406 p. ISBN 8474262186.

ARTíCULO: El futuro del hábitat: repensando la habitabilidad desde la sostenibilidad. El caso español / Joaquim Arcas-Abella, Anna Pagès-Ramon, Marina Casals-Tres 
ESPAÑA. Código Técnico de la Edificación. [En línea]. Ministerio de Fomento, Secretaría de Estado de vivienda y actuaciones urbanas. 2010. [Fecha de consulta: 17 mayo 2011] Disponible en: http://www. codigotecnico.org/web/recursos/documentos/

Código Técnico de la Edificación. Madrid, Ministerio de Vivienda, Boletín Oficial del Estado. 2006. 11 vol. ISBN 8434016311.

Congreso de los Diputados. Constitución Española. Madrid, España. 1978.

Orden de 29 de febrero de 1.944, por la que se establecen las condiciones higiénicas mínimas que han de reunir las viviendas. Boletín Oficial del Estado. (61): 1833-1834. 1944

----. Real Decreto 2429/1979, de 6 de julio, por el que se aprueba la norma básica de edificación NBECT-79, sobre condiciones térmicas en los edificios. Boletín Oficial del Estado. (253): 24524-24550. 1979.

GOUGH, lan. El enfoque de las capacidades de M Nussbaum: Un análisis comparado con nuestra teoría de las necesidades humanas. Papeles de relaciones ecosociales y cambio global. (100): 177-202 diciembre 2007. ISSN 1888-0576.

INSTITUTO Nacional de Estadística. Censos de Población y Viviendas 2001. Volumen I Resultados nacionales. Madrid, España, Instituto Nacional de Estadística. 2005. 608 p. ISBN 8426036864.

IPCC. Climate Change 2007: Mitigation of Climate Change. Contribution of Working Group III to the Fourth Assessment Report of the Intergovern- mental Panel on Climate Change. Cambridge Cambridge University Press 2007. 851 p. [En línea]. IPCC - Intergovernmental Panel on Climate Change. 2007. Disponible en: http://www.ipcc.ch/ publications_and_data/publications_ipcc_fourth_assessment_report_wg3_report_mitigation_ of_climate_change.htm

MATA, É.; LÓPEZ, F. y CUCHÍ, A. Optimization of the management of building stocks: An example of the application of managing heating systems in university buildings in Spain. Energy and Buildings. 41(12):1334-1346, diciembre 2009. ISSN 03787788. Disponible en: http://dx.doi.org/10.1016/j. enbuild.2009.07.031

MAX-NEEF, Manfred; ELIZALDE, Antonio y HOPENHAYN, Martín. Desarrollo a escala humana: conceptos, aplicaciones y algunas reflexiones. Barcelona, España, Icaria. 1994. 148 p. ISBN 8474262178

MINISTERIO de Fomento. Licencias municipales de obra (construcción de edificios) [En línea]. España Ministerio de Fomento. [Fecha de consulta: 13 marzo 2011] Disponible en: http://www.fomento. es/BE/?nivel=2\&orden $=10000000$

----. Visados de dirección de obra de los Colegios de Arquitectos Técnicos (Obras en edificación) [En línea]. España Ministerio de Fomento. [Fecha de consulta: 14 marzo 2011] Disponible en: http:// www.fomento.es/BE/?nivel=2\&orden=09000000

MONTEYS, Xavier [et al.]. Rehabitar en nueve episodios [1]. Madrid, España, Ministerio de la Vivienda. 2010. 30 p. ISBN 9788496387492.

revista invi № 72 / Agosto 2011 / Volumen № 26: 65-93 
NACIONES Unidas. Comité de Derechos Humanos. Observación general No 4 sobre el derecho a una vivienda adecuada. 1991.

Convención marco de las Naciones Unidas sobre el cambio climático. 27 p. [En línea]. Naciones Unidas. 1992. [Fecha de consulta: 2 marzo 2011]. Disponible en: http://unfccc.int/resource/docs/ convkp/convsp.pdf

----. Outcome of the work of the ad hoc working group on long-term cooperative action under the convention: Advance unedited version. Draft decision CP.16. 29 p. [En línea]. The United Nations Climate Change Conference in Cancun. 2010. [Fecha de consulta: 2 marzo 2011]. Disponible en: http:// unfccc.int/files/meetings/cop_16/application/pdf/ cop16_lca.pdf.

----. Primera Conferencia de Naciones Unidas sobre los Asentamientos Humanos (Habitat I). Vancouver, Canadá, ONU. 1976

NAREDO, José Manuel y CUCHÍ, Albert. El libro verde del medio ambiente urbano en el ámbito de la edificación. Libro verde del medio ambiente urbano. Madrid, España, Ministerio de Medio Ambiente. 2007. p. 88-112. [En línea]. Disponible en: http:// www.bcnecologia.net/documentos/libroverde.pdf

NAREDO, José Manuel. La cara oculta de la crisis: El fin del boom inmobiliario y sus consecuencias. [En línea]. Revista de economía crítica. (7):118-133, enero 2009. ISSN 1696-0866. Disponible en: http:// www.revistaeconomiacritica.org/sites/default/files/revistas/n7/6_la_cara_oculta_de_la_crisis.pdf

92 revista invi № 72 / Agosto 2011 / Volumen № 26: 65-93
----. Perspectivas de la vivienda. Información Comercial Española, ICE: Revista de economía. (815):143154, mayo 2004. ISSN 0019-977X.

NøRGARD, Jørgen S. Consumer efficiency in conflict with GDP growth. Ecological Economics. (57):1529, abril 2006. ISSN 0921-8009.

PAGÈS, Anna y CUCHÍ, Albert. Moving the entire building sector towards low $\mathrm{co} 2$ emissions. EN: PASSIVE and Low Energy Architecture 2008 Conference PLEA 2008. (25th, 2008, Dublin, Irlanda). Towards zero energy building. Dublin, Irlanda, University College Dublin, School of Architecture, Landscape and Civil Engineering. 2008. 6 p.

POLIMENI, John M.; MAYUMI, Kozo; GIAMPIETRO, Mario y ALCOTT, Blake. The myth of resource efficiency: the jevons paradox. Londres, Reino Unido, Earthscan. 2009. 200 p. ISBN 9781844078134.

PRICE, Lynn. [et al.]. Sectoral trends in global energy use and greenhouse gas emissions. [En línea]. Environmental Energy Technologies Division, Ernest Orlando Lawrence Berkeley National Laboratory. 2006. [Fecha de consulta: 2 marzo 2011]. Disponible en: http://ies.lbl.gov/iespubs/56144.pdf

REAL Academia Española. Diccionario de la lengua española. 22a edición. Madrid, España, Espasa Calpe. 2001. ISBN 978-84-239-6813-8.

SOLANAS, Toni. Vivienda y sostenibilidad en España. Vol. 2: colectiva. Barcelona, España, Gustavo Gili. 2008. 224 p. ISBN 9788425222016.

ARTíCULO: El futuro del hábitat: repensando la habitabilidad desde la sostenibilidad. El caso español / Joaquim Arcas-Abella, Anna Pagès-Ramon, Marina Casals-Tres 
WRIGLEY, E.A. Gentes, ciudades y riqueza. La transformación de la sociedad tradicional. Barcelona, España, Editorial Crítica. 1992. 476 p. ISBN 8474235286.

ZABALZA, Ignacio. Adaptación de la metodología del análisis de ciclo de vida para la evaluación y la mejora del impacto energético y ambiental de la edificación en España. Tesis doctoral, 449 p. [En línea]. Universidad de Zaragoza, Departamento de Ingeniería Mecánica. 2011. [Fecha de consulta: 17 mayo 2011] Disponible en: http://zaguan.unizar. es/record/5751/files/TESIS-2011-032.pdf 